\title{
Designing a testing device for customized hip prosthesis
}

\author{
Dan Leordean ${ }^{1}$, Florin-Andrei Purdea ${ }^{1, *}$, Mihai Țepeș ${ }^{1}$ and Andrei Vasile Valach ${ }^{1}$ \\ ${ }^{1}$ Technical University of Cluj-Napoca
}

\begin{abstract}
The practical reference of this article is part of the technical and medical field of designing, modeling and testing of hip prostheses. The purpose of the article is to present a stand that tests the hip orthopedic prostheses. After the initial design, the whole assembly was redesigned both dimensionally and functionally based on the results obtained by the finite element method. The main results from the design of the stand are both the wide range of types of hip prostheses that can be tested as well as the actual simulation of the kinematics of the joint. The simulations performed correspond to the different daily actions: walking, running and other special conditions. The practical implications of this research are the possibility of testing the time endurance of prosthesis in the laboratory condition. The results obtained from this research can be applied to the development of new generations of prostheses, starting from materials with better endurance and reaching aspects of shape and customizable optimal dimensions.
\end{abstract}

\section{Introduction}

Hip joint simulators were developed for predicting, by attempting to duplicate in vitro physiological loads and motion, the wear rate that total hip replacements are likely to show in vivo. From a theoretical point of view, loading and motion cycles of hip joints could be closely reproduced by three rotation actuators and three force actuators. However existing devices have been designed assuming that some of these degrees of freedom are negligible, in order to reduce the complexity of the equipment. The present study singles out some preliminary indications on the design choices regarding the spatial configuration of loading and motion actuators. The aim is to define theoretically a simplified simulator but still able to apply the most physiologically realistic loading cycle to the specimen [1].

The stage of development and research in this field is in a relatively incipient form, but with very rapid development. The global manufacturer that defines the required standards is Advanced Mechanical Technology Inc. (AMTI), from the USA, which has developed for the Orthopedic Innovation Center 12 simulators that can reproduce activities of daily life, such as running or climbing stairs [2].

Prior documentation was required for this device regarding product design in this particular field, medical technology $[3,4]$.

\footnotetext{
* Corresponding author: purdeaflorin@gmail.com
} 
There are several types of tests that are performed for hip implants: ASTM F2025 (Practice for Gravimetric Measurement of Polymeric Components for Wear Assessment), ISO 14242 [5] (Implants for surgery — Wear of total hip-joint prostheses), Adverse Wear Tests (Roughened femoral heads to a specified Ra value), ISO 14242-4 (Microseparation and Direct Edge Loading of Hip Prostheses), ASTM F3047M (Standard Guide for High Demand Hip Simulator Wear Testing of Hard-on-hard Articulations), ASTM F2582 (Standard Test Method for Impingement of Acetabular Prostheses), Shoulder Wear Tests (Modification of ISO 14242 for Shoulder System Wear Test), ISO 18192 (Wear of Total Intervertebral Spinal Disc Prostheses).

Joint simulator wear testing allows researchers, manufacturers, and industry professionals to evaluate the wear performance of their prosthesis design and bearing materials [6-8] under physiological conditions. These tests can help our clients optimize [9] and improve their designs prior to large-scale manufacturing and implantation, in addition to meeting regulatory requirements.

\section{Methods}

This article presents a stand/device solution for testing orthopedic hip prostheses, stand that performs all the movements that a leg performs in normal walking, but also special regimes with high loads in the maximum positions of the races.

Prosthesis testing is absolutely necessary, because these prostheses without being rigorously tested beforehand cannot be implanted in the body of a person. In order to be introduced into the human body, it is necessary to know aspects of resistance over time, of their wear and of the surfaces with which they come into contact (acetabular and femoral area).

It addresses the design of a constructive variant for all 3 axes, analyzing advantages and disadvantages for each variant, but also choosing final variants for each axis, depending on constructive variants. In the following chapter of results and discussions the article will present a finite element analysis (FEA) for the most requested benchmarks. Following the analysis the landmarks were in a walking regime, namely the flexion-extension movement, the abduction-adduction movement, respectively the inner and outer rotation, but also the loading up to $10,000 \mathrm{~N}$. It was desired that all 3 movements could be carried out simultaneously, along with the loading, in order to be able to carry out as accurate simulations of the designed regimes as possible to withstand the existing demands.

The final solution will have to solve both the kinematic movements and to satisfy the requirements imposed by the required testing standards.

The methodology used is a practical one and envisages the elaboration of three constructive variants of the kinematic axes of the hip joint (adduction/abduction, internal/external rotation, respectively flexion/extension). These variants are thoroughly analyzed by several criteria that led to a design direction.

\subsection{Design of the chassis and the adduction/abduction axis}

For the design of the chassis and the adduction/abduction axis, three constructive variants have been proposed.

The advantages for the first variant (Fig. 1 a) are:

- because the transmission of the rotation movement is done by means of gear, large forces can be used;

- the use of a pneumatic engine reduces costs.

The shortcomings of this variant are:

- the impossibility of turning with high precision;

- high costs for processing the rack and gear; 
a)
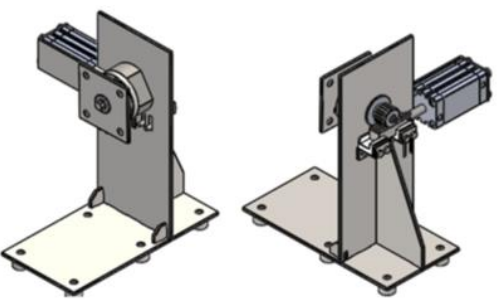

b)

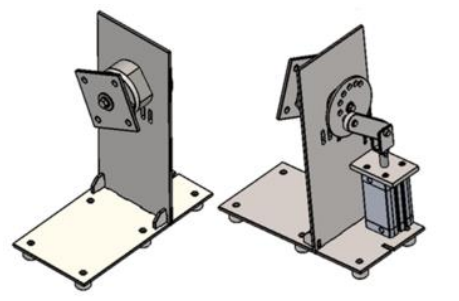

c)

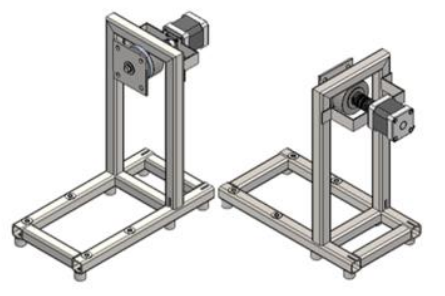

Fig. 1. Proposed variants for the design of the chassis and the adduction/abduction axis.
- large number of component elements;

- the need for additional reinforcements for the resistance structure as there is the possibility that it will to axial compression at high loads.

The second variant (Fig.1 b) has the following main advantages: the holes in the connecting rod can help us quickly adjust the walking regimes so that they can be simulated.

The shortcomings of this variant are:

- low rigidity of the resistance structure;

- $75^{\circ}$ angle stroke is more difficult realize;

- the relatively large number of component elements.

The third variant (Fig. 1 c) has the following advantages:

- the servomotor can be set to obtain accurate the required rotation of $75^{\circ}$;

- the number of component elements of the variant is significantly reduced.

The limitation of this variant are:

- high use of welded assemblies

- the cost of a servomotor is much higher than the cost of a pneumatic engine.

For the variant to be designed, the variant 2 will be combined with the one from the variant 3 , in the sense that all the operating principle from the variant 2 and the structure from the variant 3 . A linear pneumatic motor used in variant 2 will be used for actuation. It was chosen for this combination, because the servomotors are expensive and do not justify in this case the characteristics compared to those of the pneumatically driven motors. In addition to the justification it can be added that the resistance structure of variant 3 presents a number of advantages to the detriment of variants 1 and 2, of which it was mentioned: much lower processing cost, reduction of the weight of the whole subassembly, assembly of components much faster and easier.

\subsection{Design of the loading system and the rotation axis}

For the design of the loading system and the rotation axis, the variants presented in figure 2 were modeled.

The disadvantages of the first variant (Fig. 2 a) consist in that than the loading system is rigid so the shocks cannot be cushioned.

The weaknesses of the second variant (Fig. 2 b) are:

- possibility to grip the charging device;

- the way of loading the load is difficult;

- the possibility of buckling;

- high friction between the nut that performs the loading;

- the rotation is made by means of too many joints.

The third variant (Fig. 2 c) has the following shortcomings:

- high friction between nut and spring;

- the possibility of buckling the charging device;

- loading is difficult; 
a)

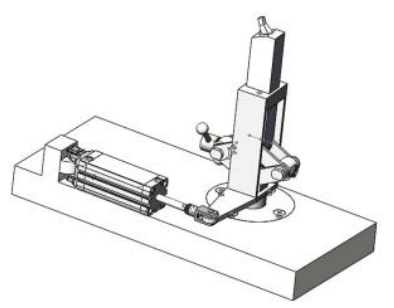

b)

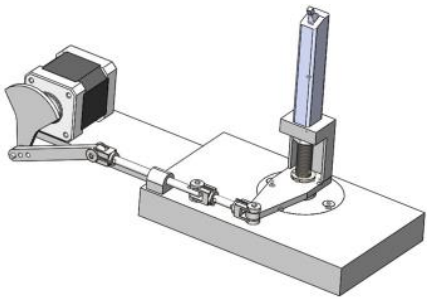

c)

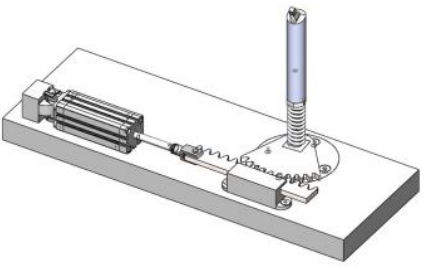

Fig. 2. Proposed variants for the design of the loading system and the axis of rotation.
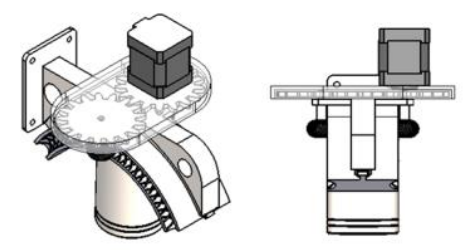

a)
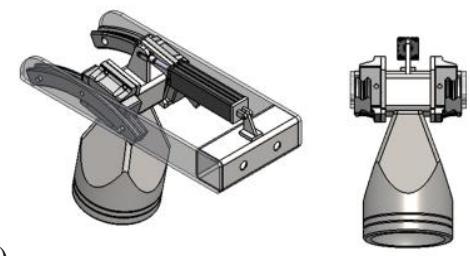

b)
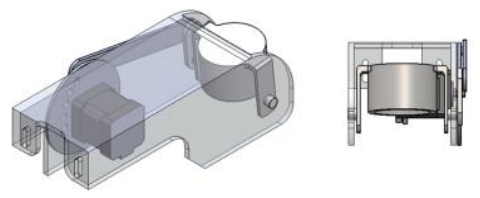

c)

Fig. 3. Proposed variants for flex/extension axis design.
- high friction between the rack and its support.

It was decided that for the design of the final version it would be combined from the first version from which loading system will be taken. The rotation of the pneumatic linear motor is done as in the third variant, the construction of the take-over of the shocks will using the spring.

\subsection{Design of the flexion/extension axis}

For the design of the flexion/extension axis, the following variants presented in figure 3 were constructed.

The disadvantages of the first variant are:

- extremely low efficiency;

- the impossibility of precise rotation;

- very low reliability.

- $120^{\circ}$ angle stroke is difficult.

The deficiency of the second variant are:

- the guides cannot be found at the dealer with a radius of less than $100 \mathrm{~mm}$;

- $120^{\circ}$ angle stroke is difficult.

The biggest disadvantage to this variant, which will lead to its exclusion from development, is the technological impossibility of realizing the alignment of the guides and the closing of the chain of dimensions between the sled and the crossbar.

In the third variant, the limitations are:

- achieving an angle of $120^{\circ}$ is difficult, because it is more difficult to symmetrical displacement with the angle required for the extension $\left(20^{\circ}\right)$;

- high use of welded assemblies.

An improvement of this variant is the use of asynchronous motors.

For the variant to be designed, the variant 2 will be combined with the variant 3 , in the sense that all the principle of operation and structure from the variant 3 will be preserved. The drive will be made with a double-acting linear pneumatic motor, used in variant 2. The authors opted for this combination, because the servomotors are relatively expensive and do not justify their characteristics to those of the pneumatically driven engines. 


\section{Results and discussions}

The most requested components of the device were analyzed with finite elements (FEA). Thus, multiple analyses were performed to improve both their geometry and their strength.

As for the shaft analysis (Fig. 4), it was decided to change the material from S128 to $\mathrm{C} 45$, it is found that the tensions are below the flow limit of the material. Next, the side plate of the chassis from the flex-extension axis assembly is analyzed.

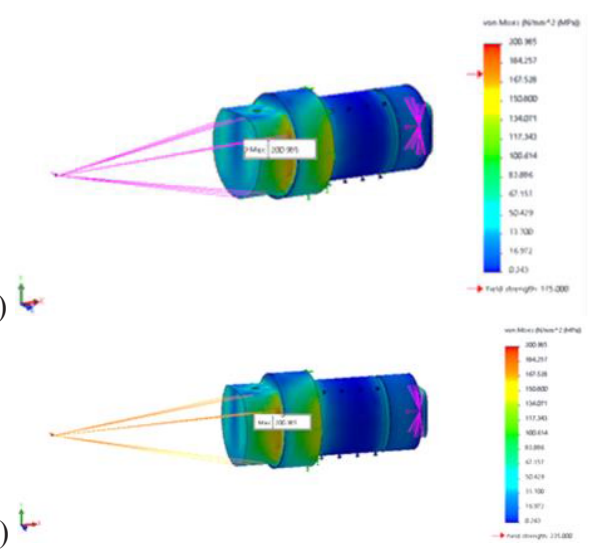

Fig. 4. The total von Mises equivalent stress for a) the initial variant and b) the redesigned version of the shaft.

According to the analysis, it can be seen that in figure 5 the total equivalent tension von Mises far exceeds the material flow limit (962MPa compared to $275 \mathrm{MPa}$, with $349 \%$ above the flow limit), which will lead us to its redesign from the original version. It was noticed that there were problems in the fixture area. The proposed solutions are to increase the thickness of the sheet from 3 $\mathrm{mm}$ to $5 \mathrm{~mm}$ and to rearrange the gaps to save material.

Following its redesign it will be observed that the model falls within the flow limit of the material (213MPa).

Regarding the base plate of loading system shown in figure 6 , by using FEA, an oversize was observed. Thus to optimize it will thin the tail of the piece. As a result of the modifications made, an increase of the total von Mises equivalent tension from $97 \mathrm{MPa}$ to $246.5 \mathrm{MPa}$ was achieved, below the material flow limit. Following the redesign have result final variants for the three subassemblies, presented in figures 7 to 9 .

After the three subassemblies were made, they were assembled to form the final product (Fig. 10). Adjustments were made to allow all axes to pass through the acetabulum axis. As a result of the changes, the movements was more efficient and their proper correlation in running regimes.

a)
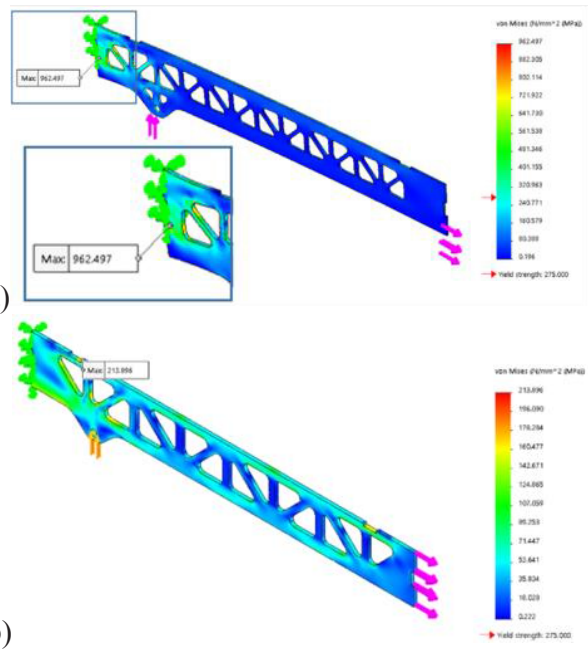

Fig. 5. The total von Mises equivalent stress for a) the initial variant and $b$ ) the redesigned version of the chassis side plate. a)
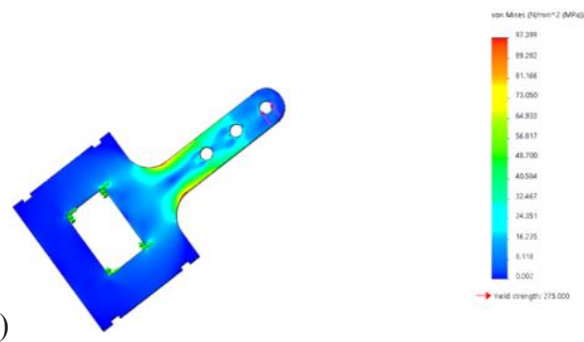

b)
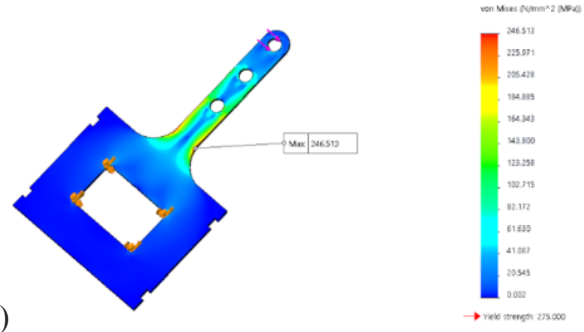

Fig. 6. The total von Mises equivalent stress for a) the initial variant and b) the redesigned version of the clamping plate. 


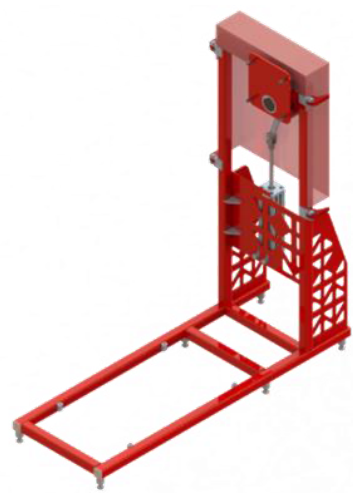

Fig. 7. The final version of the chassis and the adduction/abduction axis.

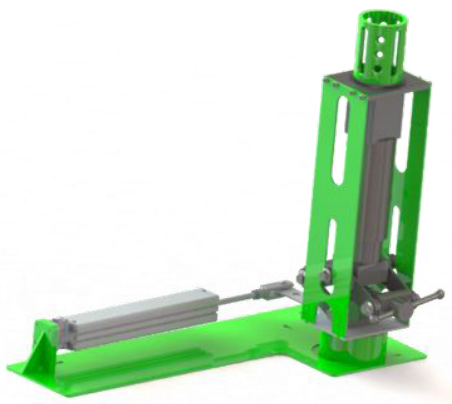

Fig. 8. Final version of the loading system and the rotation axis.

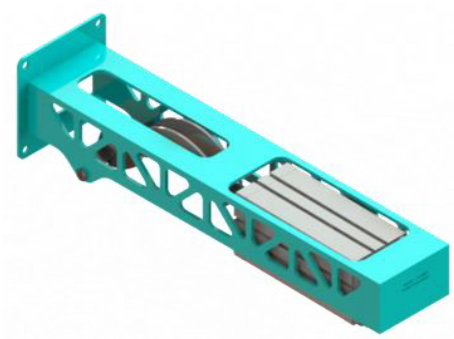

Fig. 9. Final variant of the flexion/extension axis.

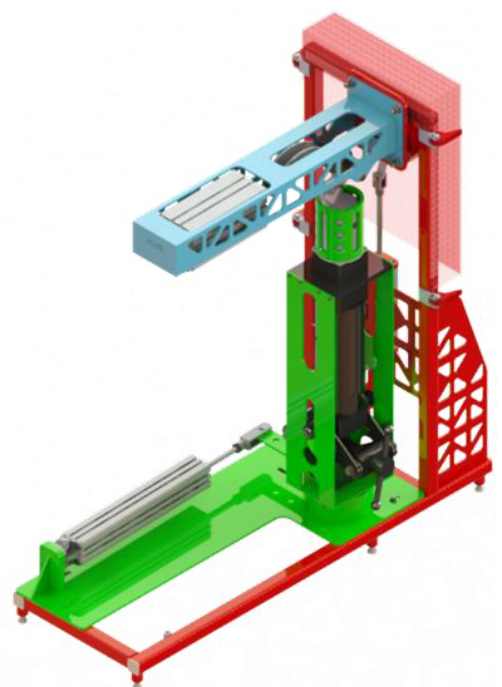

Fig. 10. Final assembly.

\section{Conclusions}

FEA has helped us to improve device benchmarks subjected to high tensions and deformations, in order to withstand for its special operating conditions. In the case of the shaft, just by modifying the material, the total amount of equivalent tension supported has decrease by $15 \%$. By redesigning the side plate, an improvement of $71.4 \%$ was achieved. The clamping plate improved by $54 \%$.

The construction of this device is simple, from the point of view of performing the movements. It is also economical with welded assemblies and pneumatic motors for transmitting movements and is conforming to ISO standards for hip implants testing.

\section{References}

1. M. Viceconti, G. Cavallotti, et al, Med. Eng.\&Phy., DOI: 10.1016/1350-4533(95)00026-7 (1996)

2. http://www.orthoinno.com/medical-device-testing/hip-simulator-wear-testing (2019)

3. A. Sarca, D. Leordean, C. Vilău, DOI: 10.4028/www.scientific.net/AMM.808.271 (2015)

4. C. Vilău, N. Balc, D. Leordean, DOI: 10.4028/www.scientific.net/AMM.808.264 (2015)

5. C. Kaddick, M. A. Wimmer, DOI: 10.1243/0954411011536019, 215 (5) (2001)

6. M. Toru, T. Yoshio, et al, J. of Orth. Resch., DOI: 10.1002/jor.22526, 32 (3), (2014)

7. R. Crockett, M. Roba, M. Naka, et al, J. of Bio. Mat. Resch. Part A, DOI: 10.1002/jbm.a.32036, 89 A (4) (2009)

8. J. Nevelos, E. Ingham, C. Doyle, et al, DOI: 10.1054/arth.2000.8100, 15 (6) (2001)

9. S.L. Bevill, G.R. Bevill, J.R. Penmetsa, et al. et al, J. of Biomechanics, DOI: 10.1016/j.jbiomech.2004.10.022, 38 (12) (2005) 